\title{
Genetic and phenotypic correlations between surrogate measures of insulin release obtained from OGTT data
}

\author{
Anette P. Gjesing • Rasmus Ribel-Madsen • Marie N. Harder • \\ Hans Eiberg • Niels Grarup • Torben Jørgensen • Claus T. Ekstrøm • \\ Oluf Pedersen • Torben Hansen
}

Received: 20 August 2014 / Accepted: 12 January 2015 /Published online: 9 February 2015

(C) Springer-Verlag Berlin Heidelberg 2015

\begin{abstract}
Aims/hypothesis We examined the extent to which surrogate measures of insulin release have shared genetic causes. Methods Genetic and phenotypic correlations were calculated in a family cohort $(n=315)$ in which beta cell indices were estimated based on fasting and oral glucose-stimulated plasma glucose, serum C-peptide and serum insulin levels. Furthermore, we genotyped a large population-based cohort $(n=$
\end{abstract}

Anette P. Gjesing and Rasmus Ribel-Madsen contributed equally to this study.

Electronic supplementary material The online version of this article (doi:10.1007/s00125-015-3516-9) contains peer-reviewed but unedited supplementary material, which is available to authorised users.

A. P. Gjesing $(\bowtie) \cdot$ R. Ribel-Madsen • M. N. Harder • N. Grarup •

O. Pedersen $\cdot T$. Hansen

The Novo Nordisk Foundation Center for Basic Metabolic Research, Section of Metabolic Genetics, Faculty of Health and Medical

Sciences, University of Copenhagen, Universitetsparken 1-3,

DK-2100 Copenhagen, Denmark

e-mail: Anette.Gjesing@sund.ku.dk

H. Eiberg

Department of Cellular and Molecular Medicine, Faculty of Health and Medical Sciences, University of Copenhagen,

Copenhagen, Denmark

T. Jørgensen

Faculty of Health and Medical Sciences, University of Copenhagen,

Copenhagen, Denmark

\section{T. Jørgensen}

Faculty of Medicine, University of Aalborg, Aalborg, Denmark

T. Jørgensen

Research Center for Prevention and Health, Glostrup, University

Hospital, Glostrup, Denmark

\section{T. Ekstrøm}

Department of Biostatistics, Faculty of Health and Medical Sciences, University of Copenhagen, Copenhagen, Denmark
$6,269)$ for common genetic variants known to associate with type 2 diabetes, fasting plasma glucose levels or fasting serum insulin levels to examine their association with various indices.

Results We found a notable difference between the phenotypic and genetic correlations for the traits, emphasising that the phenotypic correlation is an insufficient measure of the magnitude of shared genetic impact. In addition, we found that corrected insulin response, insulinogenic index and incAUC for insulin after an oral glucose challenge shared the majority of their genetic backgrounds, with genetic correlations of $0.80-0.99$. The BIGTT index for acute insulin response differed slightly more from the latter with genetic correlations of $0.78-0.87$. The HOMA for beta cell function was genetically closely related to fasting insulin with a genetic correlation of 0.85 . The effects of 82 selected susceptibility single nucleotide polymorphisms on these insulin secretion indices supported our interpretation of the data and added insight into the biological differences between the examined traits.

Conclusions/interpretation The level of shared genetic background varies between surrogate measures of insulin release, and this should be considered when designing a genetic association study to best obtain information on various mechanisms of insulin release.

Keywords Association - Diabetes · Genetic correlation · Genetics · Insulin secretion

$\begin{array}{ll}\text { Abbreviations } & \\ \rho \mathrm{E} & \text { Environmental correlation } \\ \rho \mathrm{G} & \text { Genetic correlation } \\ \rho \mathrm{P} & \text { Phenotypic correlation } \\ \mathrm{AIR} & \text { Acute insulin response } \\ \text { BIGTT-AIR BIGTT } & \text { Index for acute insulin response } \\ \mathrm{CIR} & \text { Corrected insulin response } \\ \text { HOMA-B } & \text { HOMA for beta cell function }\end{array}$


IncAUC

SNP

Incremental AUC

Single nucleotide polymorphism

\section{Introduction}

Type 2 diabetes is a complex disease that results from the interaction of multiple genetic and environmental factors affecting insulin secretion and sensitivity. It has been demonstrated that the heritability of insulin secretion is high compared with other intermediate phenotypes associated with type 2 diabetes [1, 2]. Studies aiming to characterise the genetic background for beta cell defects typically use physiological measures that reflect the early phase of the dynamic beta cell response to a glucose challenge. In studies of smaller wellcharacterised populations, expensive and laborious in vivo methods, such as IVGTT, can be applied, whereas large genetic epidemiological studies use surrogate indices based on fasting blood samples or an OGTT. The HOMA for beta cell function (HOMA-B) represents a simple equation to estimate the percentage of remaining beta cell function from fasting values of plasma insulin and glucose, and this index has been validated against IVGTT data [3]. The insulinogenic [4] and the corrected insulin response (CIR) [5] indices have been established to assess the first phase insulin secretory capacity after an oral glucose load independent of the plasma glucose level, whereas the BIGTT index for acute insulin response (BIGTT-AIR) is mathematically modelled to simulate the first phase beta cell response to intravenous glucose from OGTT data supplemented by information on sex and BMI [6]. Often, the investigators of a specific study include one or a few of these indices, and the choice of traits may be based on observed differences in phenotypic correlations. The phenotypic correlation is, however, a measure covering both genetic and environmental influences, whereas traits for a genetic epidemiological study should ideally be selected to distinguish differences in genetic contribution alone.

The present study aims to examine the phenotypic and genetic correlations of fasting and OGTT-derived indices describing beta cell function in a family-based study sample. Furthermore, in a large population-based cohort, the effects of single nucleotide polymorphisms (SNPs) known to associate with type 2 diabetes, fasting plasma glucose levels, 2-h plasma glucose levels or fasting serum insulin levels were examined, applying various beta cell indices.

\section{Methods}

Study populations The study population for the investigation of genetic correlations consisted of 533 individuals from 95 families with one parent suffering from verified type 2 diabetes according to WHO 1999 criteria [7] and one parent without known diabetes. All non-diabetic family members (spouses, offspring and other relatives) were asked to participate and, among these, 366 individuals underwent an OGTT. Sixtythree families having four or more children were included in the correlation analysis comprising a total of 315 non-diabetic individuals (Table 1). Furthermore, 292 of these individuals also underwent an IVGTT. Prior to participation, informed consent was obtained from all participants. The study was approved by the Ethical Committee of Copenhagen (KA 93033 and KA 93033gm) and was conducted in accordance with the principles of the Declaration of Helsinki II.
Table 1 Clinical characteristics of study populations

Data are median (interquartile range) or mean $\pm \mathrm{SD}$

\begin{tabular}{|c|c|c|}
\hline Trait & Families & Inter99 \\
\hline$n$ (female/male) & $315(134 / 181)$ & $5,776(2,898 / 2,878)$ \\
\hline Age, years & $40.63(34.35 ; 50.81)$ & $45(40 ; 50)$ \\
\hline BMI, kg/m² & $25.51(22.72 ; 28.63)$ & $25.6(23.2 ; 28.5)$ \\
\hline Fasting plasma glucose $(\mathrm{mmol} / \mathrm{l})$ & $5.2 \pm 0.6$ & $5.5 \pm 0.8$ \\
\hline Fasting serum insulin, pmol/1 & $35(24 ; 51)$ & $34(24 ; 51)$ \\
\hline Fasting serum C-peptide, pmol/1 & $485.3(397.8 ; 619.3)$ & $534.0(534.0 ; 709.8)$ \\
\hline Serum insulin $30 \mathrm{~min}, \mathrm{pmol} / 1$ & $271(184 ; 381)$ & $246(175 ; 354)$ \\
\hline HOMA-B, \% & $436(320 ; 634)$ & $355(248 ; 518)$ \\
\hline AUC insulin, $\mathrm{pmol} / 1 \times \min$ & $25,510(18,190 ; 36,410)$ & $23,200(16,810 ; 33,160)$ \\
\hline IncAUC insulin, $\mathrm{pmol} / 1 \times \min$ & $21,170(15,120 ; 31,220)$ & $18,700(13,060 ; 27,370)$ \\
\hline IncAUC C-peptide, $\mathrm{pmol} / 1 \times \min$ & $115,700(90,660 ; 144,900)$ & $220,000(178,100 ; 274,800)$ \\
\hline Insulinogenic index & $79.8(53.4 ; 114.4)$ & $71.5(44.7 ; 119.2)$ \\
\hline CIR & $112.2(72.0 ; 179.4)$ & $93(57 ; 152)$ \\
\hline BIGTT-AIR & $2,328(1,795 ; 3,034)$ & $1,627(1,284 ; 2,078)$ \\
\hline AIR (IVGTT), nmol/1× min & $1,644(1,043 ; 2,494)$ & - \\
\hline
\end{tabular}


Specific SNPs associating with type 2 diabetes and glucose metabolic traits were examined in the Danish populationbased Inter99 cohort, which included 6,269 middle-aged people without known type 2 diabetes (www.ClinicalTrials.gov no. NCT00289237, www.inter99.dk, Table 1).

Clinical examination All participants underwent measures of height and weight for calculation of BMI, and a WHO-defined and standardised OGTT was conducted in individuals without known type 2 diabetes. Fasting blood samples were drawn after $12 \mathrm{~h}$ of fasting followed by a $75 \mathrm{~g}$ glucose load and blood sampling at 30 and $120 \mathrm{~min}$ for analysis of plasma glucose, serum insulin and serum C-peptide. In addition, IVGTT was performed after a 12 -h fast. Venous blood samples were drawn from the contralateral antecubital vein at fasting and at 2, 3, 4, $5,6,7,8,10,12,14,16$ and $19 \mathrm{~min}$ for analysis of plasma glucose, serum insulin and serum C-peptide.

Calculation of surrogate measures of insulin release Surrogate measures of insulin release included in the present study were HOMA-B [3] based on fasting blood samples and as insulinogenic index [4], CIR [5], incremental AUC (incAUC) for insulin and C-peptide ( $t=0,30$ and $120 \mathrm{~min})$ and BIGTT-AIR [6] based on the OGTT, and for comparison (AIR) calculated as the incAUC for insulin from $0-8 \mathrm{~min}$ following an IVGTT. Indices were calculated according to the formulas shown in Electronic Supplementary Material (ESM) Table 1.

Genotyping Participants from the family $(n=378)$ and Inter99 $(n=6,377)$ cohorts were genotyped by the Metabochip on an Illumina HiScan (Illumina, San Diego, CA, USA). Genotypes were called using the Genotyping module (version 1.9.4) of GenomeStudio software (version 2011.1, Illumina) and custom cluster data were generated from 6,000 Danish DNA samples analysed on the same HiScan. Quality control to remove closely related individuals and individuals with an extreme inbreeding coefficient was applied to data from Inter99. Successful genotyping was accomplished among 5,739 individuals from Inter99. In the families, participants were excluded in the case of disagreement between questionnaire information on relationship and actual genotype resemblance. Individuals with a low call rate, those with a mislabelled sex and individuals with a high discordance rate to previously genotyped SNPs were also excluded in both populations. Successful genotyping was accomplished among 343 individuals.

Genetic and environmental correlations Genetic, phenotypic and environmental correlations were calculated using the software package SOLAR (http://solar.txbiomedgenetics.org, version 4.2.0) that performs statistical genetic analyses for family-based data using variance components [8]. The genetic correlations are measures of the additive effects of shared genes in bivariate analysis using the command 'polygenic'. Residuals were examined for univariate normality prior to each analysis and traits were inverse normal transformed to satisfy the assumption of normality. The effect of the shared environment was investigated in the univariate analyses by use of the 'house' command. However, none of the traits were significantly affected and thus this variable was not included in subsequent analyses (ESM Table 2). We allowed for correlations between both the genetic residual additive components for each trait and the non-shared environmental correlations for each trait adjusted for sex and age as well as sex, age and variants significantly influencing both of the traits included in the bivariate analyses. This was achieved by including sex, age and variants as covariates in the analysis. However, correlations with BIGTT-AIR were only adjusted for age, since sex is included in the calculations of the BIGTT-AIR index. Table 2 presents the genetic vs the phenotypic correlations, whereas ESM Table 3 presents the genetic vs the environmental correlations.

SNP associations to glucose metabolic traits Eighty-two SNPs previously shown to associate with type 2 diabetes, fasting glucose, fasting insulin or 2-h glucose in genomewide association studies of Europeans were selected from the Metabochip [9]. Additive genetic models of each SNP adjusted for sex and age were established for associations with HOMA-B, BIGTT-AIR, insulinogenic index and CIR (ESM Table 1) using the glm command in R (www.r-project.org, version 3.0.1). The response variable was transformed by natural logarithm if not normally distributed.

\section{Results}

Genetic correlation Phenotypic $(\rho \mathrm{P})$, genetic $(\rho \mathrm{G})$ and environmental $(\rho \mathrm{E})$ correlations were calculated in a family-based study population for the traits representing beta cell function in the fasting state and in response to oral or intravenous glucose loads (Table 2 and ESM Table 3).

The traits showing the highest level of genetic correlation were insulinogenic index and CIR $(\rho \mathrm{G}=0.99 \pm 0.02)$. Insulinogenic index and CIR were also highly genetically correlated to other measures of stimulated insulin secretion (incAUC insulin: $\rho \mathrm{G}=0.80 \pm 0.16$ and $\rho \mathrm{G}=0.80 \pm 0.18$, respectively; AIR: $\rho \mathrm{G}=0.87 \pm 0.10$ and $\rho \mathrm{G}=0.80 \pm 0.10$, respectively) but not with stimulated C-peptide (incAUC C-peptide: $\rho \mathrm{G}=0.47 \pm 0.22$ and $\rho \mathrm{G}=0.35 \pm 0.22$, respectively). The environmental correlation was also strong between CIR and insulinogenic index $(\rho \mathrm{E}=0.95 \pm 0.01)$, however, the environmental correlations of CIR and insulinogenic index with incAUC insulin $(\rho \mathrm{E}=0.38 \pm 0.10$ and $\rho \mathrm{E}=0.62 \pm 0.07$, respectively) and $\operatorname{AIR}(\rho \mathrm{E}=0.64 \pm 0.16$ and $\rho \mathrm{E}=0.59 \pm 0.17$, respectively) were modest. 
Table 2 Phenotypic and genetic correlations for insulin secretion traits in the family population

\begin{tabular}{|c|c|c|c|c|c|c|c|c|c|}
\hline & HOMA-B & BIGTT-AIR & $\begin{array}{l}\text { Insulinogenic } \\
\text { index }\end{array}$ & CIR & $\begin{array}{l}\text { IncAUC } \\
\text { insulin }\end{array}$ & $\begin{array}{l}\text { IncAUC } \\
\text { C-peptide }\end{array}$ & $\begin{array}{l}\text { Fasting serum } \\
\text { insulin }\end{array}$ & $\begin{array}{l}\text { Fasting serum } \\
\text { C-peptide }\end{array}$ & AIR (IVGTT) \\
\hline HOMA-B & & $\begin{array}{l}0.81(0.09) \\
p_{1}=0.0002 \\
p_{2}=0.0011\end{array}$ & $\begin{array}{l}0.59(0.20) \\
p_{1}=0.03 \\
p_{2}=0.01\end{array}$ & $\begin{array}{l}0.60(0.17) \\
p_{1}=0.01 \\
p_{2}=0.001\end{array}$ & $\begin{array}{l}0.81(0.13) \\
p_{1}=0.002 \\
p_{2}=0.04\end{array}$ & $\begin{array}{l}0.42(0.19) \\
p_{1}=0.06 \\
p_{2}=0.0000015\end{array}$ & $\begin{array}{l}0.85(0.08) \\
p_{1}=0.001 \\
p_{2}=0.02\end{array}$ & $\begin{array}{l}0.33(0.22) \\
p_{1}=0.2 \\
p_{2}=0.00005\end{array}$ & $\begin{array}{l}0.66(0.13) \\
p_{1}=0.0003 \\
p_{2}=0.0005\end{array}$ \\
\hline BIGTT-AIR & 0.74 & & $\begin{array}{l}0.83(0.11) \\
p_{1}=0.004 \\
p_{2}=0.03\end{array}$ & $\begin{array}{l}0.87(0.08) \\
p_{1}=0.0008 \\
p_{2}=0.008\end{array}$ & $\begin{array}{l}0.78(0.14) \\
p_{1}=0.01 \\
p_{2}=0.04\end{array}$ & $\begin{array}{l}0.26(0.23) \\
p_{1}=0.3 \\
p_{2}=0.00005\end{array}$ & $\begin{array}{l}0.53(0.21) \\
p_{1}=0.07 \\
p_{2}=0.004\end{array}$ & $\begin{array}{l}0.02(0.27) \\
p_{1}=0.9 \\
p_{2}=0.00001\end{array}$ & $\begin{array}{l}0.85(0.09) \\
p_{1}=0.000001 \\
p_{2}=0.02\end{array}$ \\
\hline $\begin{array}{l}\text { Insulinogenic } \\
\text { index }\end{array}$ & 0.55 & 0.75 & & $\begin{array}{l}0.99(0.02) \\
p_{1}=0.0005 \\
p_{2}=0.4\end{array}$ & $\begin{array}{l}0.80(0.16) \\
p_{1}=0.03 \\
p_{2}=0.07\end{array}$ & $\begin{array}{l}0.47(0.22) \\
p_{1}=0.09 \\
p_{2}=0.01\end{array}$ & $\begin{array}{l}0.35(0.30) \\
p_{1}=0.3 \\
p_{2}=0.006\end{array}$ & $\begin{array}{l}-0.008(0.30) \\
p_{1}=1.0 \\
p_{2}=0.009\end{array}$ & $\begin{array}{l}0.87(0.10) \\
p_{1}=0.00002 \\
p_{2}=0.1\end{array}$ \\
\hline CIR & 0.58 & 0.81 & 0.96 & & $\begin{array}{l}0.80(0.18) \\
p_{1}=0.008 \\
p_{2}=0.1\end{array}$ & $\begin{array}{l}0.35(0.22) \\
p_{1}=0.1 \\
p_{2}=0.0002\end{array}$ & $\begin{array}{l}0.47(0.25) \\
p_{1}=0.1 \\
p_{2}=0.01\end{array}$ & $\begin{array}{l}-0.01(0.26) \\
p_{1}=1.0 \\
p_{2}=0.0004\end{array}$ & $\begin{array}{l}0.80(0.10) \\
p_{1}=0.00002 \\
p_{2}=0.01\end{array}$ \\
\hline IncAUC insulin & 0.62 & 0.64 & 0.66 & 0.50 & & $\begin{array}{l}0.43(0.22) \\
p_{1}=0.2 \\
p_{2}=0.01\end{array}$ & $\begin{array}{l}0.60(0.21) \\
p_{1}=0.1 \\
p_{2}=0.007\end{array}$ & $\begin{array}{l}0.11(0.31) \\
p_{1}=0.7 \\
p_{2}=0.009\end{array}$ & $\begin{array}{l}0.65(0.17) \\
p_{1}=0.005 \\
p_{2}=0.04\end{array}$ \\
\hline IncAUC C-peptide & 0.41 & 0.42 & 0.44 & 0.31 & 0.71 & & $\begin{array}{l}0.23(0.25) \\
p_{1}=0.4 \\
p_{2}=0.002\end{array}$ & $\begin{array}{l}0.66(0.14) \\
p_{1}=0.002 \\
p_{2}=0.00004\end{array}$ & $\begin{array}{l}0.17(0.19) \\
p_{1}=0.4 \\
p_{2}=0.000002\end{array}$ \\
\hline $\begin{array}{l}\text { Fasting serum } \\
\quad \text { insulin }\end{array}$ & 0.81 & 0.53 & 0.48 & 0.39 & 0.68 & 0.45 & & $\begin{array}{l}0.60(0.17) \\
p_{1}=0.04 \\
p_{2}=0.003\end{array}$ & $\begin{array}{l}0.37(0.21) \\
p_{1}=0.1 \\
p_{2}=0.005\end{array}$ \\
\hline $\begin{array}{l}\text { Fasting serum } \\
\text { C-peptide }\end{array}$ & 0.55 & 0.33 & 0.31 & 0.22 & 0.46 & 0.58 & 0.75 & & $\begin{array}{l}0.04(0.21) \\
p_{1}=0.9 \\
p_{2}=0.000008\end{array}$ \\
\hline AIR (IVGTT) & 0.48 & 0.66 & 0.62 & 0.65 & 0.47 & 0.30 & 0.29 & 0.14 & \\
\hline
\end{tabular}

Genetic correlation estimated as the $\rho \mathrm{G}(\mathrm{SE})$ is presented in the upper right part of the table, whereas phenotypic correlations estimated as the $\rho \mathrm{P}$ are presented in the lower left part of the table

$p_{1}, p$ value for test of $\rho \mathrm{G} \neq 0 ; p_{2}, p$ value for test of $\rho \mathrm{G} \neq 1$

Fig. 1 Schematic illustration of genes in which variants are showing either exclusively or overlapping associations at $p<0.05$ with CIR, insulinogenic index, BIGTT-AIR and/or HOMA-B based on association analyses in 5,739 individuals

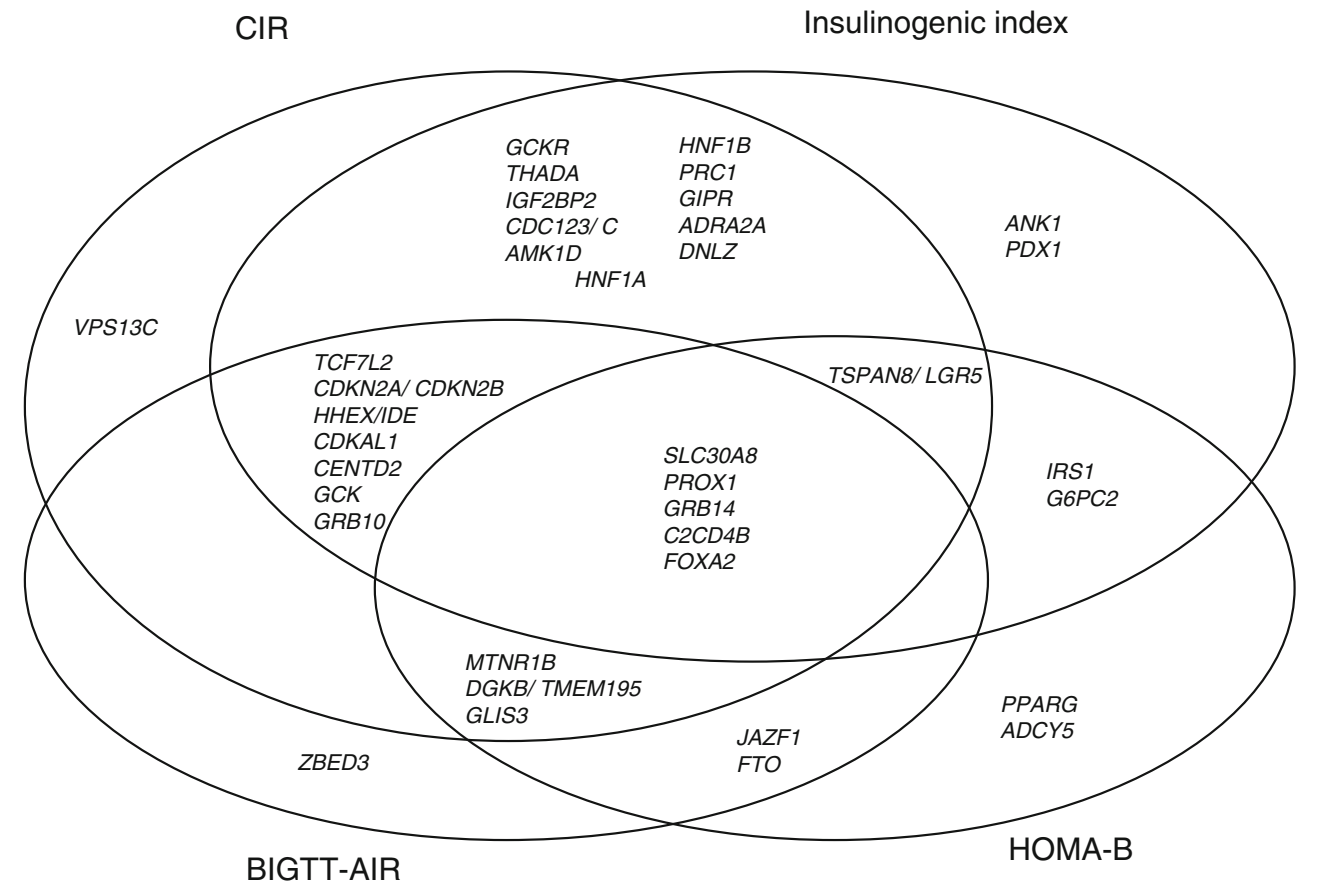


BIGTT-AIR was highly genetically correlated with CIR ( $\rho \mathrm{G}=0.85 \pm 0.09)$ and despite high genetic correlations with the remaining measures of stimulated insulin secretion (insulinogenic index, incAUC insulin and AIR), these were significantly different from complete genetic correlation. Genetic correlations with fasting $(\rho \mathrm{G}=0.02 \pm 0.27)$ and stimulated C-peptide ( $\rho \mathrm{G}=0.26 \pm 0.23$ ) were very low.

HOMA-B displayed a high genetic correlation with fasting insulin $(\rho \mathrm{G}=0.85 \pm 0.08)$ and incAUC insulin $(\rho \mathrm{G}=0.81 \pm$ $0.13)$, but a lower correlation with fasting C-peptide $(\rho \mathrm{G}=$ $0.46 \pm 0.18)$ and incAUC C-peptide $(\rho \mathrm{G}=0.42 \pm 0.19)$. Adjusting the genetic correlation between HOMA-B and incAUC insulin and incAUC C-peptide, respectively, for fasting levels of insulin and C-peptide, reduced the genetic correlations ( $\rho \mathrm{G}=0.65 \pm 0.23$ and $\rho \mathrm{G}=0.21 \pm 0.20$, respectively). HOMA-B was also strongly environmentally correlated with fasting insulin $(\rho \mathrm{E}=0.82 \pm 0.05)$, but not with incAUC insulin $(\rho \mathrm{E}=0.54 \pm 0.09)$.

IncAUC for insulin and C-peptide showed a modest genetic correlation $(\rho \mathrm{G}=0.43 \pm 0.22)$ despite a strong environmental correlation $(\rho \mathrm{E}=0.92 \pm 0.06$; ESM Table 3$)$.

Genetic associations In order to understand how the indices of insulin secretion reflect different physiological mechanisms, we tested the effects of known type 2 diabetes susceptibility and glycaemic trait variants in a population-based study population (Inter99) with insulin secretion indices measured, including CIR, insulinogenic index, HOMA-B and BIGTT-AIR (Fig. 1 and ESM Table 4). Ten SNPs associated with both CIR and insulinogenic index, but no other indices, and seven SNPs associated with CIR, insulinogenic index and BIGTT-AIR. By contrast, only five SNPs associated with CIR, insulinogenic index, BIGTT-AIR and HOMA-B, two SNPs associated with both HOMA-B and insulinogenic index, and two SNPs associated with both HOMA-B and BIGTT-AIR. None of the variants associated exclusively with CIR and HOMA-B, insulinogenic index and BIGTT-AIR or BIGTT-AIR and CIR. Thus, we found a large overlap with traits showing a high degree of genetic correlation in the family study and traits having a large number of shared associating variants in the population-based cohort.

Some indices associated specifically with one of the selected SNPs and out of the 82 SNPs examined, six associated with only one trait. VPS13C rs17271305 was the only SNP associating specifically with CIR and ZBED3 rs4457053 associated exclusively with BIGTT-AIR. ANK1 rs516946 and PDX1 rs2293941 associated exclusively with insulinogenic index, and PPARG rs 1801282 and ADCY5 rs11708067 associated with HOMA-B.

Genetic correlation adjusted for genetic associations Subsequently, we investigated how much of the shared genetics could be explained by the 82 common SNPs identified so far. Thus, the genetic correlation calculated in the family cohort was adjusted for the variants found in the populationbased cohort to associate with both of the traits included in the bivariate analyses. However, none of the genetic correlations were reduced by this adjustment (data not shown).

\section{Discussion}

In the present study, we used family pedigrees to examine genetic correlations for a number of surrogate measures of insulin secretion that are typically used in large genetic epidemiological studies.

Genetic correlation Overall, it was found that the majority of the genetic correlations between insulin secretory measures exceeded the corresponding phenotypic correlations. However, several traits did not display complete genetic correlation and thus comprise some residual variation that likely reflects partly different genetic backgrounds for insulin secretion.

Among the traits under almost identical genetic control were insulinogenic index, CIR and incAUC insulin. BIGTTAIR also showed a high level of genetic correlation with insulinogenic index, CIR and incAUC insulin, however, it was not complete $(\rho \mathrm{G} \neq 1)$. Yet, these four measures of insulin secretion were only slightly genetically correlated with fasting C-peptide and incAUC C-peptide, which is surprising considering that $\mathrm{C}$-peptide is secreted equimolarly with insulin.

However, C-peptide does not undergo hepatic first-pass metabolism [10], and the lack of genetic correlation between measures of C-peptide and insulin may indicate that measures of insulin are influenced not only by genes involved in insulin secretion but also by genes involved the removal of insulin from the circulation.

HOMA-B was, as expected, strongly genetically correlated with fasting serum insulin.

Genetic association Two genetic variants exclusively influenced HOMA-B. Namely, the type 2 diabetes risk allele of PPARG rs1801282 previously found to associate with insulin resistance $[11,12]$ and the $A D C Y 5$ variant previously found to associate with birthweight and fasting glucose apart from HOMA-B [13, 14]. The major allele of G6PC2 rs560887 was associated with decreased HOMA-B as well as increased insulinogenic index. This variant has in previous studies been found to be associated with elevated fasting glucose [15] and it likely regulates the expression of G6PC2 encoding the catalytic subunit of glucose-6-phosphatase, a regulator of glycolytic flux in the beta cell. The major allele is proposed to cause a rightward shift of the dose-response curve for the glucosestimulated insulin secretion [16], which explains the 
association with HOMA-B and insulinogenic index in opposite directions.

Type 2 diabetes- and fasting glucose-associated variants in or near VPS13C, ANK1 and PDX1 associated exclusively with either CIR or insulinogenic index. Knowledge on their role in glucose metabolism is currently too limited to propose molecular mechanisms distinguishing CIR and insulinogenic index from each other [17-19]. The 2-h glucose-raising GIPR rs10423928 associated with both CIR and insulinogenic index, but not with HOMA-B or BIGTT-AIR that are expected to reflect primarily basal and glucose-stimulated insulin secretion, respectively. GIPR encodes the receptor for glucosedependent insulinotropic polypeptide located on the beta cell, thus CIR and the insulinogenic index seem to capture variations influencing the effect of incretin on beta cell function. The type 2 diabetes susceptibility variant $G C K$ rs 4607517 , on the other hand, associated with CIR, insulinogenic index and BIGTT-AIR in accordance with the known function of glucokinase function as a hexokinase crucial to glucose-stimulated insulin release. Five SNPs associated with all of the surrogate measures of insulin secretion. Among these were SLC30A8 rs3802177 encoding the ZnT8 zinc transporter important to insulin crystallisation in the secretory granules in the beta cells [20], which exemplifies that a defect at the level of granule formation will affect both the fasting and stimulated beta cell response to glucose and incretins. Hence, based on the genetic correlations and association studies, our data suggest that BIGTT-AIR is influenced by genes involved in incretinindependent dynamic glucose-induced insulin secretion, whereas insulinogenic index and CIR in addition are influenced by genes involved in incretin-stimulated insulin secretion.

Adjusting the genetic correlations for common SNPs associating significantly with type 2 diabetes and glucose metabolic traits had little influence, suggesting these variants explain only a fraction of the common genetic influences on the traits investigated. This finding is not surprising considering the minor effect sizes of these variants and may indicate that low frequent or rare variants are responsible for the majority of shared genetic influence between these traits.

The genetic correlation is a measure of the shared genetics between two traits and may exist for two reasons: (1) due to pleiotropy between traits, meaning that the same genes are influencing multiple phenotypic traits, e.g. through a common pathway; or (2) due to gene-gene interactions, where the impact of one gene is influenced by another (set of) gene(s). The traits included in the present study are closely related as they are based on the same raw values of fasting and stimulated glucose and insulin measurements. Thus, we assume that the genetic correlation in the present study is mainly a result of the traits sharing biological pathways.

In conclusion, the shared genetic background varies between surrogate measures of insulin release, and the majority of the different measures of insulin secretion did not show complete genetic correlations. Thus, they likely represent slightly different contributions to the genetics of insulin secretion, which should be considered when designing genetic association studies to best obtain information on various mechanisms of insulin release.

Acknowledgements The authors thank A. Forman, B. Andersen, G. J. Klavsen, T. H. Lorentzen and M. Modest for their dedicated and careful technical assistance, and G. Lademann and A. L. Nielsen for secretarial and administrative support (all from The Novo Nordisk Foundation Center for Basic Metabolic Research, Section of Metabolic Genetics, University of Copenhagen).

Funding The study was supported by grants from the Danish Medical Research Council; the Danish Council for Independent Research; the University of Copenhagen; an EEC grant (BMH4CT950662); the Velux Foundation; the Lundbeck Foundation Centre of Applied Medical Genomics for Personalized Disease Prediction and Care (LUCAMP; www. lucamp.org); the European Foundation for the Study of Diabetes (EFSD) and the Danish Diabetes Association. The Novo Nordisk Foundation Center for Basic Metabolic Research is an independent Research Center at the University of Copenhagen partially funded by an unrestricted donation from the Novo Nordisk Foundation (www.metabol.ku.dk).

Duality of interest TH and OP hold personal shares in Novo Nordisk. All other authors declare that there is no duality of interest associated with their contribution to this manuscript.

Contribution statement APG, RRM, MNH, CTE, NG, HE, OP, TJ and TH contributed to the conception and design of the study. APG, RRM, MNH, CTE, NG, OP and TH contributed to the analysis and interpretation of data. The first manuscript was written by APG and RRM with equal contribution and the final draft was finalised by APG, RRM, MNH, CTE, NG, HE, OP, TJ and TH. All authors have revised the manuscript critically for important intellectual content and approved the final version of the paper. $\mathrm{TH}$ is the guarantor of this work.

\section{References}

1. Poulsen P, Levin K, Petersen I, Christensen K, Beck-Nielsen H, Vaag A (2005) Heritability of insulin secretion, peripheral and hepatic insulin action, and intracellular glucose partitioning in young and old Danish twins. Diabetes 54:275-283

2. Gjesing AP, Ekstrom CT, Eiberg $H$ et al (2012) Fasting and oral glucose-stimulated levels of glucose-dependent insulinotropic polypeptide (GIP) and glucagon-like peptide-1 (GLP-1) are highly familial traits. Diabetologia 55:1338-1345

3. Matthews DR, Hosker JP, Rudenski AS, Naylor BA, Treacher DF, Turner RC (1985) Homeostasis model assessment: insulin resistance and beta-cell function from fasting plasma glucose and insulin concentrations in man. Diabetologia 28:412-419

4. Seltzer HS, Allen EW, Herron AL Jr, Brennan MT (1967) Insulin secretion in response to glycemic stimulus: relation of delayed initial release to carbohydrate intolerance in mild diabetes mellitus. J Clin Invest 46:323-335

5. Sluiter WJ, Erkelens DW, Reitsma WD, Doorenbos H (1976) Glucose tolerance and insulin release, a mathematical approach I. Assay of the beta-cell response after oral glucose loading. Diabetes $25: 241-244$ 
6. Hansen T, Drivsholm T, Urhammer SA et al (2007) The BIGTT test: a novel test for simultaneous measurement of pancreatic beta-cell function, insulin sensitivity, and glucose tolerance. Diabetes Care 30:257-262

7. Alberti KG, Zimmet PZ (1998) Definition, diagnosis and classification of diabetes mellitus and its complications. Part 1: diagnosis and classification of diabetes mellitus provisional report of a WHO consultation. Diabet Med 15:539-553

8. Almasy L, Blangero J (1998) Multipoint quantitative-trait linkage analysis in general pedigrees. Am J Hum Genet 62:1198-1211

9. Voight BF, Kang HM, Ding J et al (2012) The metabochip, a custom genotyping array for genetic studies of metabolic, cardiovascular, and anthropometric traits. PLoS Genet 8:e1002793

10. Polonsky KS, Rubenstein AH (1984) C-peptide as a measure of the secretion and hepatic extraction of insulin. Pitfalls and limitations. Diabetes 33:486-494

11. Trombetta M, Bonetti S, Boselli ML et al (2013) PPARG2 Pro12Ala and ADAMTS9 rs4607103 as "insulin resistance loci" and "insulin secretion loci" in Italian individuals. The GENFIEV study and the Verona Newly Diagnosed Type 2 Diabetes Study (VNDS) 4. Acta Diabetol 50:401-408

12. Tellechea ML, Aranguren F, Perez MS, Cerrone GE, Frechtel GD, Taverna MJ (2009) Pro12Ala polymorphism of the peroxisome proliferator activated receptor-gamma gene is associated with metabolic syndrome and surrogate measures of insulin resistance in healthy men: interaction with smoking status. Circ J 73:2118-2124
13. Horikoshi M, Yaghootkar H, Mook-Kanamori DO et al (2013) New loci associated with birth weight identify genetic links between intrauterine growth and adult height and metabolism. Nat Genet 45:76-82

14. Florez JC, Jablonski KA, McAteer JB et al (2012) Effects of genetic variants previously associated with fasting glucose and insulin in the Diabetes Prevention Program. PLoS ONE 7:e44424

15. Dupuis J, Langenberg C, Prokopenko I et al (2010) New genetic loci implicated in fasting glucose homeostasis and their impact on type 2 diabetes risk. Nat Genet 42:105-116

16. Pound LD, Oeser JK, O'Brien TP et al (2013) G6PC2: a negative regulator of basal glucose-stimulated insulin secretion. Diabetes 62:1547-1556

17. An CH, Kim YR, Kim HS, Kim SS, Yoo NJ, Lee SH (2012) Frameshift mutations of vacuolar protein sorting genes in gastric and colorectal cancers with microsatellite instability. Hum Pathol 43:40-47

18. Bennett V, Baines AJ (2001) Spectrin and ankyrin-based pathways: metazoan inventions for integrating cells into tissues. Physiol Rev 81:1353-1392

19. Gannon M, Ables ET, Crawford L et al (2008) pdx-1 function is specifically required in embryonic beta cells to generate appropriate numbers of endocrine cell types and maintain glucose homeostasis. Dev Biol 314:406-417

20. Lemaire K, Ravier MA, Schraenen A et al (2009) Insulin crystallization depends on zinc transporter ZnT8 expression, but is not required for normal glucose homeostasis in mice. Proc Natl Acad Sci U S A 106:14872-14877 